\title{
IMPLEMENTATION OF A CHUA CIRCUIT TO DEMONSTRATE BifurCations and Strange AtTractors in a Class
}

\author{
Ahmad Byagowi $^{1}$ and Witold Kinsner ${ }^{1,2,3}$ \\ ${ }^{1}$ Department of Electrical and Computer Engineering \\ University of Manitoba, Winnipeg, MB, Canada R3T 5V6 \\ and ${ }^{2}$ The Institute of Industrial Mathematical Sciences \\ and ${ }^{1}$ Telecommunications Research Laboratories, TRLabs \\ \{byagowi@cc.umanitoba.ca|kinsner@ece.umanitoba.ca\}
}

\begin{abstract}
This paper describes the design and implementation of a Chua double-scroll circuit to demonstrate chaos in dynamical systems to students in a graduate course in order to enhance their visualization and understanding of strange attractor and Feigenbaum bifurcation trees.

Teaching dynamical systems (i.e., nonlinear systems that can exhibit chaos) is often considered difficult because of the mathematical modeling involved and the inclusion of the fourth strange-attractor state, in addition to the traditional point stability, cyclic stability, and toroidal stability, as found in dynamic systems. A graduate course has been offered at the University of Manitoba for many years to provide both (i) a unified theory of fractal dimensions, together with many practical implementations of algorithms to compute the fractal dimensions, including the Rényi dimension spectrum that is required for characterization of the strange attractors using multifractal analysis.

Leon Chua developed a simple nonlinear circuit capable of producing a rich collection of dynamic phenomena, ranging from fixed points to cycle points, standard bifurcations (period doubling), other standard routes to chaos, and chaos itself. The reason for selecting this specific circuit as a class demonstration tool is threefold: (i) the circuit has an analytical model and can be simulated, (ii) the circuit is implementable using available commercial off-the-shelf components, and (iii) the signals in the circuit can be acquired without affecting and altering its operation significantly.

This paper describes the architecture, implementation, verification, and testing of the Chua system, as well as an analysis of the data obtained during the current phase of the development. Although there are many possible implementations of Chua's circuit, our implementation has several innovative design features to make it more applicable to enhance students' learning in the classroom.
\end{abstract}

Keywords: Chaos; chaos visualization; bifurcation diagram; Chua circuit; multi-tap digital potentiometer.

\section{INTRODUCTION}

\subsection{Usefulness of Demonstrations in Class}

Teaching undergraduate and graduate engineering students in a classroom environment usually involves a number of techniques, including oral presentations and discussions, visual materials (images and video) to augment difficult ideas, written homework, quizzes tests, and exams, as well as hands-on laboratories. Classroom demonstrations of existing artefacts (chips, processors, boards, electromechanical devices, subsystems and systems) may also be very effective. Of particular importance are demonstrations developed specifically for the course by course participants, as they understand the challenges in acquiring difficult material.

In the case of dynamical systems (i.e., nonlinear dynamic systems that can exhibit chaos), the material taught requires considerable sophistication from the students, and explanations by traditional techniques may not be adequate [Kins12]. Consequently, using demonstrations of real examples of dynamical systems can ease the understanding of the underlying concepts, particularly when the demonstrations can behave in a fully-controlled fashion.

There are many such examples of dynamical systems that can be used for demonstration. However, there are only a few nonlinear systems that satisfy the following three key requirements simultaneously: (i) they can be described by an appropriate mathematical model (e.g., differential equations), can be modelled on a computer, and (iii) can be implementable in the laboratory for classroom demonstrations and research. Such examples should also satisfy additional requirements, including: (i) be inexpensive to implement with commercial off-theshelf (COTS) components, (ii) easy to maintain, (iii) easy to modify, (iv) durable in multiple use, and should be easy to monitor without affecting the quality of the measured signal (dynamical systems are sensitive to initial conditions and thus to sensor probes). 


\section{BACKground ON ChuA Circuit}

\subsection{The Chua Circuit as a Dynamical System}

Around 1983, Leon Chua developed a nonlinear circuit (today known as the Chua circuit) that is capable of demonstrating a rich collection of dynamical phenomena, ranging from fixed points to cycle points, standard bifurcations (period doubling), other standard routes to chaos, and chaos itself (e.g., [Chua07], [Chua92], [ChPW95], [Chua06], [PaCh89], [Kapi02], [Ogor97], [Deva92], [Ott93], [AlSY96], [PeJS92], [Strog94], [BrHT10], [MaSi05], [OtSY94], [Kins12]). A few years later, Chua, Komuro, and Matsumoto proved rigorously that the Chua attractor is chaotic indeed [ChKM86]. Moreover, the circuit can meet all the requirements outlined in the previous section.

As shown in Fig. 1, the Chua circuit consists of three energy-storing components (two capacitors $C 1$ and $C 2$, one inductor $L$ ), one resistor $R$ (or equivalently conductance $G=1 / R$ ), and one nonlinear resistor $r_{\mathrm{N}}$ or conductance $g_{\mathrm{N}}$ such as the Chua diode shown in Fig. 2. This non-linear element provides the required energy for the circuit. Notice that we use capital letter to denote constant values, and lower-case letters to denote variables and values that are not constants in time. The circuit has two loops: the nonlinear loop with $C 1$ (on the left) and the purely reactive loop with $C 2$ (on the right). The two loops are coupled by the resistor $R$. When $R$ is very large, the coupling is weak, and it is strong when $R$ is small. The nonlinear resistor $r_{\mathrm{N}}$ and the coupling between the two loops are responsible largely for making the behaviour of this simple circuit extraordinary.

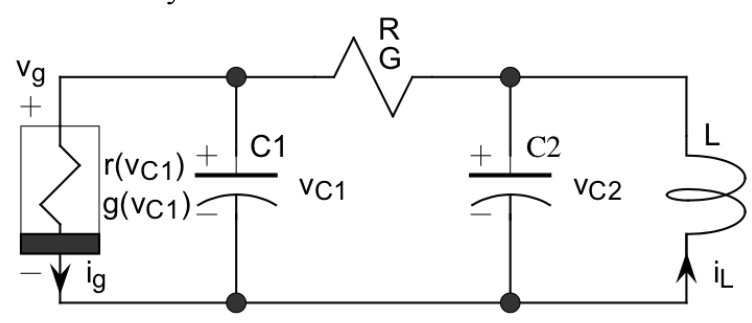

Fig. 1 Chua circuit with two capacitors ( $\mathrm{C} 1$ and $\mathrm{C} 2)$ one inductor $(\mathrm{L})$, one resistor $(\mathrm{R})$, and one nonlinear resistor

$$
\left(r_{N}\right) \text {. (From [Kins12]) }
$$

The circuit satisfies all the three requirements. First, the circuit can be modelled using standard circuit techniques.

Second, the circuit is implementable, maintainable, and can be constructed using COTS components. The behaviour of the circuit depends exclusively on the values of its components. Modification of the circuit can be achieved by either using different discrete-valued components, or variable components.
Third, since the chaotic behaviour occurs in the electric voltage and current loops in the circuit, the circuit can be sensed (monitored) by using either laboratory instruments such as an oscilloscope, or it can be captured using a standard high-impedance data acquisition instruments. Since the resistor is the only component which has a time-independent relation between voltage and current (V-I plot), changing the resistor value in the Chua circuit can be considered as the best way to modify the behaviour of the circuit.

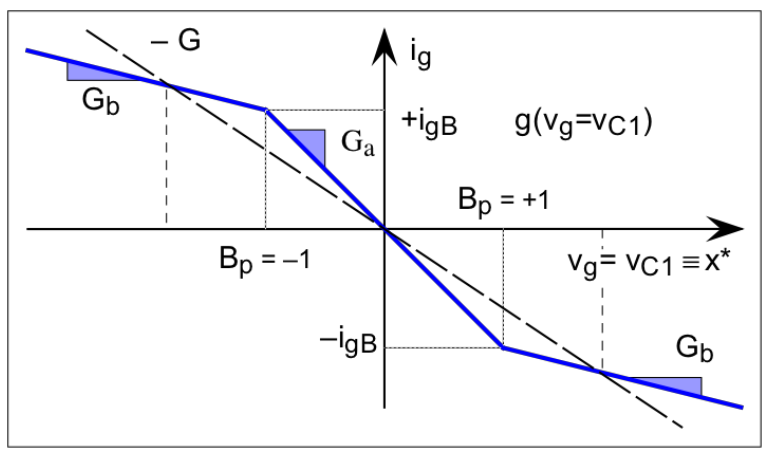

Fig. 2 Negative resistor in the Chua circuit.

(From [Kins12])

\subsection{Circuit Model}

The circuit can be modelled as a third-order RLC circuit with four linear elements and one nonlinear element. From Kirchhoff's law, one can write the following set of three first-order equations [Kapi02, Sec. 6.4], [Kins12; Ch1, Part3]

$$
\left\{\begin{aligned}
\frac{d v_{C 1}}{d t} & =\frac{1}{R C_{1}}\left(v_{C 2}-v_{C 1}\right)-\frac{1}{C_{1}} g\left(v_{C 1}\right) \\
\frac{d v_{C 2}}{d t} & =\frac{1}{R C_{2}}\left(v_{C 1}-v_{C 2}\right)+\frac{1}{C_{2}} i_{L} \\
\frac{d i_{L}}{d t} & =-v_{C 2}
\end{aligned}\right.
$$

where the nonlinear conductance is approximated by a three-segment piecewise antisymmetrical linear function (Fig. 2), with slopes $G_{a}$ (in the middle section) and $G_{b}$ (the outer sections), and the breakpoints at $B_{p}= \pm 1$

$$
g\left(x^{*} \equiv v_{g}\right)=G_{b} x *+\frac{1}{2}\left(G_{a}-G_{b}\right)\left[\left|x^{*}+B_{p}\right|-\left|x *-B_{p}\right|\right]
$$

This autonomous dynamical system (i.e., a system that evolves through its natural dynamics, without any 
external signal injection into the circuit) can be further simplified into the following dimensionless form

$$
\left\{\begin{array}{l}
\frac{d x}{d t}=\alpha(y-x-h(x)) \\
\frac{d y}{d t}=y-x+z \\
\frac{d i_{L}}{d t}=-\beta y
\end{array}\right.
$$

where

$$
\begin{cases}x=\frac{v_{C 1}}{B_{p}} & y=\frac{v_{C 2}}{B_{p}} \quad z=\frac{i_{L}}{B_{p} G} \\ \tau=\frac{t G}{C_{2}} & \\ a=\frac{G_{a}}{G} & b=\frac{G_{b}}{G} \\ \alpha=\frac{C_{2}}{C_{1}} & \beta=\frac{C_{2}}{L G^{2}}\end{cases}
$$

and

$$
h(x)=b x+\frac{1}{2}(a-b)[|x+1|-|x-1|]
$$

Notice that $x$ is related to the voltage on the $C 1, y$ relates to the voltage on $C 2$, and $z$ represents the current flowing through the inductor. The critical parameters are the ratios of the conductance of the nonlinear resistor in its middle $G_{a}$ to the coupling conductance $G$, and the outer conductance $G_{b}$ to $G$. The other parameters $\alpha$ and $\beta$ relate the value of the capacitor $C 2$ to $C 1$ and to $L$, respectively.

The circuit displays distinct classes of behaviour for different combinations of $\alpha, \beta, a$, and $b$ [Kins12]. For example, the full double-scroll chaos develops for $\alpha=$ $10.0, \beta=14.87, a=-1.27$, and $\mathrm{b}=-0.68$.

\subsection{Other Chaotic Circuits}

In addition to the original Chua circuit, there are many of its variations. For example, lattices of such circuits produce more complex behaviour.

There are also many similar simple chaotic circuits. For example, Sprott introduced "jerk" chaotic circuits described by a single third-order autonomous ordinary differential equation, with various nonlinearities, often represented by piecewise linear approximations for ease of robust electronic implementation with diodes and opamps [Spro00a], [Spro00b]. These circuits are also scalable over a wide range of frequencies.

\section{Chua Circuit Hardware Design}

\subsection{Alternative Designs}

Several alternative implementations for the Chua circuit have been considered for the demo in the fractal and chaos course [Kins12]. The selected circuit provides flexibility in order to demonstrate different states of the Chua circuit, and can provide detailed control over the behaviour of circuit.

Using a single multi-tap digital potentiometer instead of the constant resistor used in the Chua circuit, a flexible embodiment of the circuit has been implemented. The digital potentiometer can be set either manually by the user, or automatically by a microcontroller. Since the change of value of the potentiometer is automated, different control schemes can be implemented in order to adjust the behaviour of the Chua circuit. In addition, by changing the value of the resistor repeatedly, the transition of states (period doubling) in the Chua circuit can be observed clearly, and the entire bifurcation diagram can be demonstrated.

\subsection{The Chua Diode}

Since the nonlinear element $r_{N}$ does not exist as a passive component, it must be implemented using active elements such as operational amplifiers (op-amps). As there are many implementations for the Chua circuit, there are many alternative implementations of the nonlinear element (also known as the Chua diode).

Figure 3 shows the schematic of a popular hardware implementation of the Chua circuit with a dual op-amp implementation of the nonlinear Chua diode.

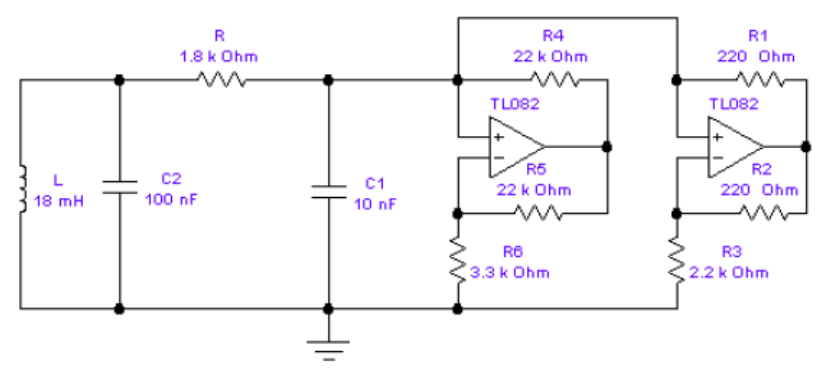

Fig. 3. Hardware implementation used for the Chua circuit.

(From [Kenn92])

The Chua diode has been implemented by many authors (e.g., [ElKe00], [Cros03]), but a robust circuit was first developed by Kennedy [Kenn92], [KeRS00]. A good discussion of op-amp related to such circuits can be found in, for example, [HoHi89, Ch. 4], [SeSm09], [NaSe03]. 


\subsection{Isolated Power Supply}

The implementation of the Chua circuit shown in Fig 3 is based on a dual op-amp chip (TL082CN) which requires a dual power supply of $\pm 15 \mathrm{~V}$. Moreover, the Chua circuit produces a wide range of frequencies that can interfere with the digital components used in the circuit. For example, the digital potentiometer is based on electronic circuits to imitate impedance variability, and requires stringent voltage conditions from a power supply. Thus, the robustness of the circuit could be increased by isolating the power supply from the digital potentiometer and its control circuit. This was done by a DC-to-DC converter that converts a single 5-V supply to $\pm 15 \mathrm{~V}$ to provide the voltage for the op-amps. The DCP0205-15DP has been selected for this purpose, and its block diagram is shown in Fig. 4 [Burr99].

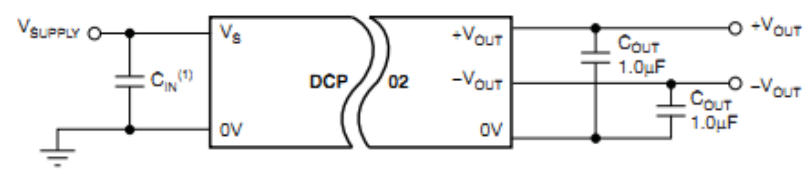

Fig. 4. DDCP0205-15DP DC-to-DC converter.

\subsection{Multi-Tap Digital Potentiometer}

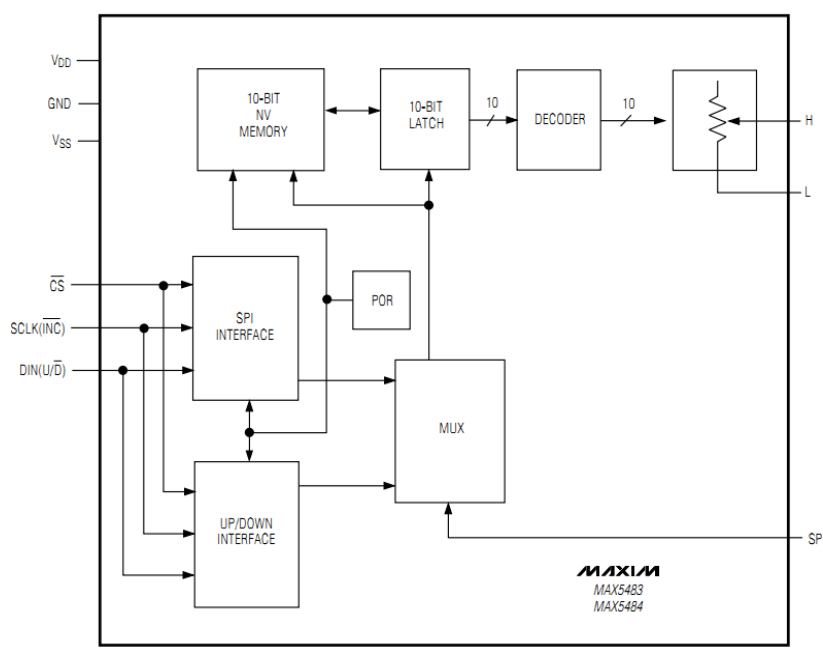

Fig. 5. MAX5483 digital potentiometer.

(From [Maxi10])

The behaviour of the Chua circuit depends on all the values of its reactive, passive and active components. In order to demonstrate the bifurcation diagram on an oscilloscope, the behaviour of the Chua circuit should repeat from the first bifurcation point $p_{0}$ to the last bifurcation point $p_{\infty}$ gradually and repeatedly. In this work, the coupling resistor $R$ is replaced with a multi-tap digital potentiometer to vary the behaviour of the circuit. A multi-tap (1024-tap) digital potentiometer (MAX5483 from MAXIM) is selected [Maxi10]. A block diagram for this component is shown in Fig. 5.

\subsection{Microcontroller}

The basic Chua circuit requires no computer, or microprocessor, or microcontroller. Our automated Chua circuit described in this paper requires a microcontroller such as the PIC16F876A [Micr03]. This controller has been selected because of much experience and support available at the University of Manitoba. Many other embedded microcontrollers could also be used to perform the required functions.

One of the functions is to control the digital potentiometer. The pot receives commands through the serial peripheral interface (SPI) bus, according to a specific protocol. The microcontroller sends the values of the required resistance of digital potentiometer between two set points over a desired time, thus producing a sawtooth waveform, as shown in Fig. 6.

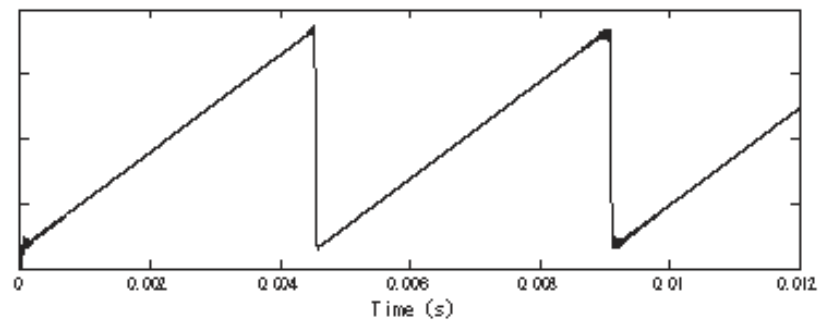

Fig. 6. Sawtooth signal used to vary the value of the potentiometer.

The sawtooth waveform is implemented using software code on the PIC16F876A microcontroller. The microcontroller increases the value of its register. When the value of the register reaches the high set point then it places the value of the low set point on the register. The incrementing repeats in order to provide the periodic waveform within a range as required by the specific mode of operation of the Chua circuit. The two set points are found in order to set the Chua circuit varying between $p_{0}$ and $p_{\infty}$. The implementation of the sawtooth waveform generator is first done in the $\mathrm{C}$ language, and then the code is compiled and programmed in the microcontroller, using the software package provided for the PIC microcontrollers.

\subsection{Breadboard Implementation}

In order to ease the implementation, a breadboard has been selected for realization of the Chua circuit and the digital controller section. 


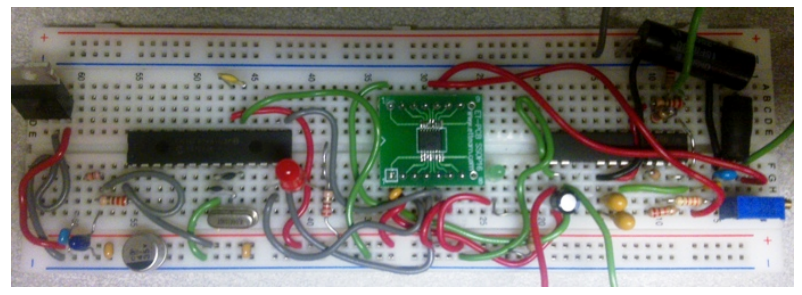

Fig. 7. Hardware implementation of the design.

Figure 7 shows the Chua circuit on the left side of the photograph, and the microcontroller is on the right side. The digital potentiometer is in the middle of the breadboard. Several LED indicators have also been installed to indicate the operation of the circuit. The values of the components used for the Chua circuit, excluding the Chua diode implementation are shown in Table 1.

Table 1. Value of components used in the Chua circuit implementation.

\begin{tabular}{|l|c|}
\hline \multicolumn{1}{|c|}{ Component } & Value \\
\hline Inductor $\mathrm{L}$ & $18 \mathrm{mH}$ \\
\hline Capacitor $\mathrm{C} 1$ & $10 \mathrm{nF}$ \\
\hline Capacitor $\mathrm{C} 2$ & $100 \mathrm{nF}$ \\
\hline Resistor $\mathrm{R}$ & $1 \mathrm{k} \Omega$ to $2 \mathrm{k} \Omega$ \\
\hline
\end{tabular}

\section{EXPERIMENTAL RESUltS}

\subsection{Bifurcation Diagram}

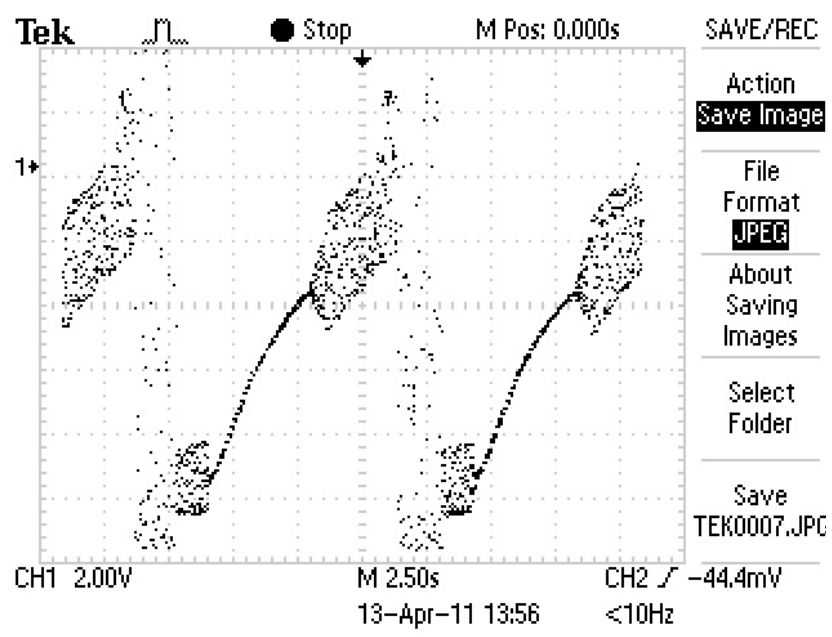

Fig. 8. Bifurcation diagram captured on a digital oscilloscope (Tektronix TDS1012B).

In any course on dynamical systems, the bifurcation diagram is one of the most important visualization tools. If a bifurcation diagram exists, the underlying process may lead to chaos. This circuit was designed to demonstrate the bifurcation diagram on either a storage oscilloscope, or through any suitable data acquisition unit. This bifurcation demonstration constitutes the first main objective of this experiment.

Figure 8 shows a screen shot of a repeated bifurcation diagram on a digital oscilloscope (Tektronix TDS1012B using its built-in screen shot function).

In order to obtain a higher resolution of the bifurcation diagram, the resistor-ramping period of the digital potentiometer is set to 10 seconds. In other words, the resistor of the Chua circuit is wiped from the low set point toward the high set point within 10 seconds.

Figure 9 shows a better resolution bifurcation diagram in order to obtain more details. This has been achieved by decreasing the time axis parameter of the oscilloscope from $2.5 \mathrm{sec}$ to $1 \mathrm{sec}$ per screen grid unit.

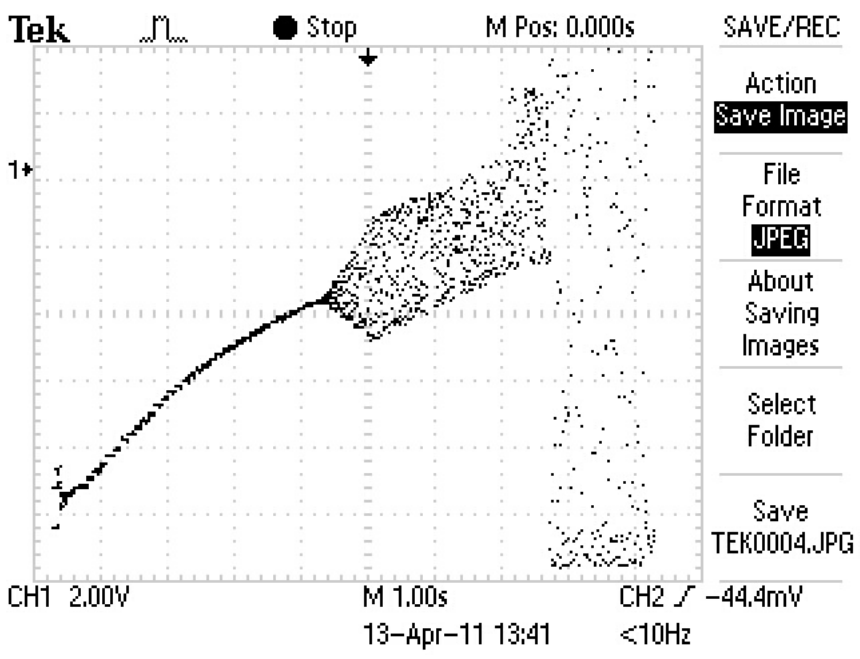

Fig. 9. A higher-resolution bifurcation diagram.

It is seen from Fig. 9 that the left side of the screenshot represents a single (1-period) solution which increases monotonically up to the first bifurcation in the middle of the screen. The band increases up to the threequarters of the screen where it spreads to the entire screen. The chaotic regime is indicated by the dots spread over the band.

\subsection{Displaying Chua Strange Attractor in 2D}

The second main objective of this experiment is to demonstrate that the Chua circuit has a strange attractor (this name was coined by Ruelle and Takens in 1971 [RuTa71]). If the attractor exists, the process cannot be random [Kins12]. Since the Chua circuit has three degrees of freedom, its state space is threedimensional (3D). One way to display a strange attractor is to take a pair of the state variables (e.g., $x$ and $y$ ), and pass them to the two deflectors $(X, Y)$ of an oscilloscope. For example, Fig. 10 shows a $2 \mathrm{D}$ projection of the $3 \mathrm{D}$ state space of the Chua circuit, while it is in the doublescroll strange attractor mode. 


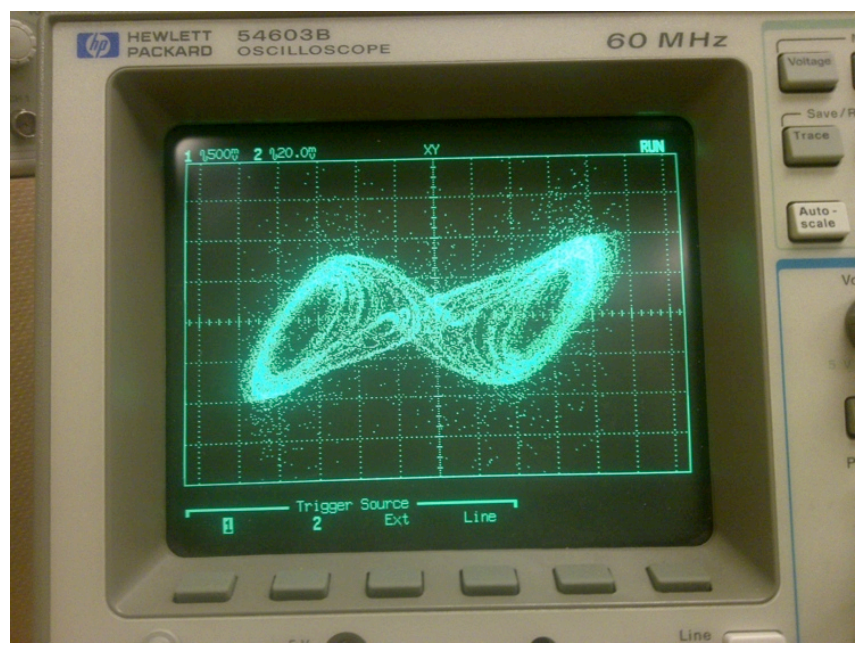

Fig. 10. Double-scroll strange attractor obtained from the Chua circuit (HP54603B).

It is seen that the strange attractor is a double-scroll indeed. Although any pair of the state variables could be used to display the strange attractor, variables $x$ and $y$ represent the voltages on the capacitors that can be tapped easily.

\subsection{Displaying Chua Strange Attractor in 3D}

Since an oscilloscope cannot display all the three dimensions of the actual attractor, the 3D state space of the circuit can be reconstructed from the three $x, y, z$ signals, as acquired from the Chua circuit, using a data acquisition unit and a computer. The data acquisition selected for this experiment is the NI-DAQCard6062E card from National Instruments [NaIn05], as shown in Fig. 11. The card can sample at a rate of up to 500 kilosamples per second $(\mathrm{kS} / \mathrm{s})$ and 12-bit resolution. An SCB068 terminal box is also used to reduce noise and signal distortion.

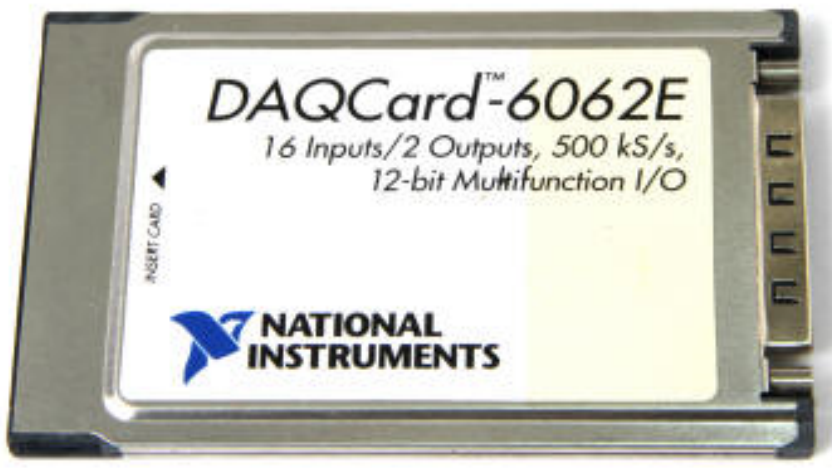

Fig. 11. Data acquisition used to reconstruct the three dimensional state space.
This data acquisition system records 10 seconds of data on all the three channels $(x, y, z)$ from the Chua circuit, and stores the data on a PC using MatLab Simulink (MDL) [NaIn08]. In order to capture the highest quality of the broadband signals generated by the Chua circuit, the highest available bandwidth of the data acquisition has been employed (50,000 samples per second, with 8-bit resolution). The record length is $5,165,229$ bytes $(4.92 \mathrm{MiB})$. At this rate, a $4 \mathrm{GiB} \mathrm{SD}$ card can store over 2.5 hours $(8,130 \mathrm{~s})$ of data.

Figure 12 shows a screen shot of a window of the captured data representing the three state variables of the Chua circuit. The screen shot has been obtained, using a built-in function on the Tektronix TDS2014B digital storage color oscilloscope. The three channels shown in Fig. 12 are the electric voltage on capacitor $C 1$ (Ch. 1), the voltage on capacitor $C 2$ (Ch. 2) and the electric current through inductor $L$ (Ch. 3 ) are shown.

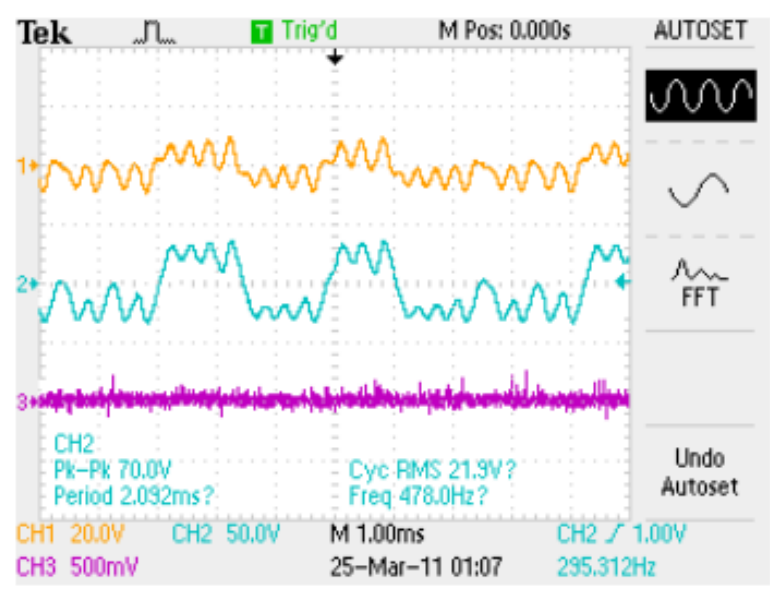

Fig. 12. Triple channel data obtained from the Chua circuit.

The above $(x, y, z)$ data acquired from the Chua circuit have then been displayed as a 3D plot, using Mathworks Maltab [Math12], and the plot is shown in Fig. 13.

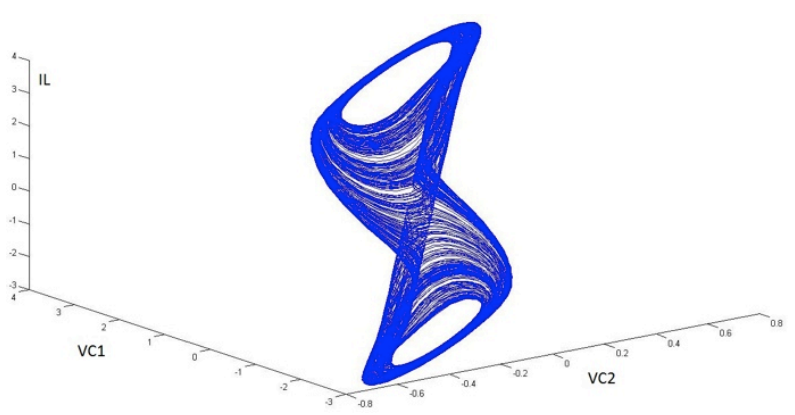

Fig. 13. Three dimensional double scroll stage attractor of the Chua circuit. 
Notice that although the plot has been rotated with respect to the oscilloscope display of Fig. 10, the double scroll character of the object is unchanged. The order of the channels might have been reversed when plotting the diagrams.

\subsection{Reconstruction of Strange Attractor}

The third main objective of this experiment is to reconstruct the strange attractor from a single time series acquired from the Chua circuit.

2D Reconstruction: A 2D projection of the strange attractor can be reconstructed from a single signal observation, by plotting the current sample of the signal $u[n+1]$ against the previous sample of the signal $u[n]$. Figure 14 shows the $2 \mathrm{D}$ strange attractor reconstructed from the $\mathrm{C} 1$ capacitor signal voltage $\left(v_{C 1}\right)$.

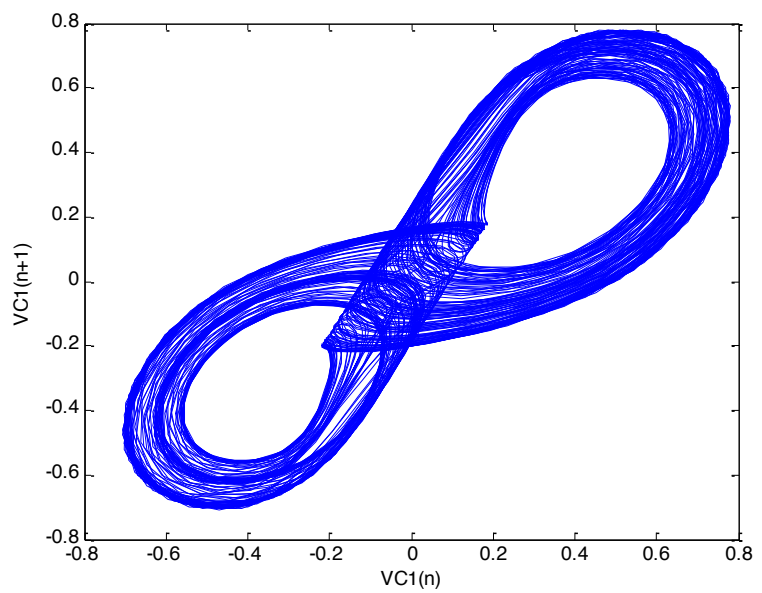

Fig. 14. 2D reconstructed strange attractor from VC1.

This result reconstructed from a single time series $v_{\mathrm{C} 1}$ is remarkable as it resembles the strange attractor displayed on the oscilloscope (Fig. 10), as generated by two separate signals, $v_{\mathrm{C} 1}$ and $v_{\mathrm{C} 2}$. Since the strange attractor has an unambiguous non-random shape, the underlying process cannot be random. The only limitation of the above 2D graph is that the double scroll intersects itself.

3D Reconstruction: The above attractor reconstruction process can be extended to the entire 3D strange attractor by plotting the signal samples with one and two time delays, $u[n+2]$ against $u[n+1]$ against $u[n]$. Figure 15 shows a $3 \mathrm{D}$ plot of the strange attractor, also reconstructed from $v_{C 1}$. This reconstructed $3 \mathrm{D}$ strange attractor is very similar to the strange attractor assembled from the three real data captured by the data acquisition.

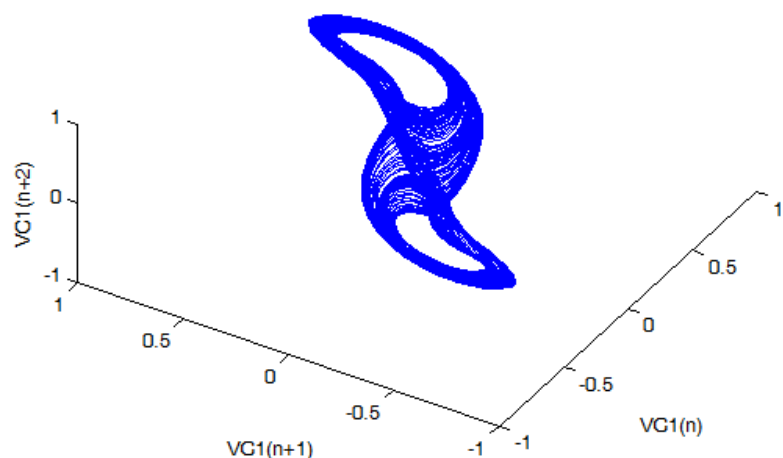

Fig. 15. 3D strange attractor reconstructed from $\mathrm{vC} 1$.

Is there anything special about the voltage on $C 1$ that allow us to reconstruct the $3 \mathrm{D}$ strange attractor with such high fidelity? The answer is clearly negative. Since each single observation time series contains the same information about the entire dynamical system, the strange attractor could be reconstructed from the other two observations, either the voltage from $C 2, v_{C 2}$, or the current through $L, i_{L}$.

\subsection{Reconstruction of Unknown Attractors}

In the case of Chua circuit, we know that its phase space has three dimensions. Thus, the reconstructed Chua strange attractor must "live" in three dimensions. We say that the embedding dimension of the attractor is $D_{E}=3$.

Takens (e.g., [Take81], [Take10]) has provided a remarkable theorem that states: a strange attractor, if one exists, can be reconstructed from a single time series, even if we do not know the number of embedding dimensions $D_{E},=m$ and the time lag $\tau$ between samples to be involved in the reconstruction. To clarify this statement, let us consider an original time series with $N$ quantized samples denoted by

$$
\{u[n]\} \quad n=1,2,3, \ldots, N
$$

The strange attractor could be reconstructed by placing its successive points on the trajectory in $m$-dimensional pseudo phase space. Each point $U[n]$ on the trajectory has $m$ coordinates selected from the original time series according to

$$
\begin{gathered}
U[n]=(u[n], u[n+\tau], u[n+2 \tau], \ldots, u[n+(m-1) \tau]) \\
\text { for } n=1,2,3, \ldots, N-(m-1)
\end{gathered}
$$

There are several good techniques to find the integer embedding dimension $D_{E}$, and the time lag $\tau$ (e.g., [Addi97], [Will97], [SaYC91]). Since both parameters 
must be estimated from the time series itself, the strange attractor now "lives" in a pseudo phase space.

One of the best techniques for finding $\tau$ is the firstminimum mutual information criterion [Will97; Ch. 19]. A good technique for finding $D_{E}$ is the false nearest neighbourhood distance criterion [Addi97]. The principle behind this technique is that if the embedding dimension of the pseudo-phase space $D_{P}$ is too small, or if $D_{P}<D_{E}$, the distances between the "false" neighbours change. However, when $D_{P} \geq D_{E}$, the distances between the "true" neighbours stop changing.

\section{VERIFICATION OF RESULTS}

To verify that the sampling frequency of $50 \mathrm{kS} / \mathrm{s}$ was sufficient for this Chua circuit, the three signals $\left(v_{C 1}, v_{C 2}\right.$, $i_{L}$ ) were checked for stationarity (the first and second moments only), then a spectral analysis was performed to establish the signal range, and the Nyquist frequency was calculated.

Each channel was checked for weak stationarity over the entire record length by calculating the first two moments (mean and variance) for three windows of sizes $100,1,000$, and 10,000 samples, with $50 \%$ overlap.

The signal ranges were also computed from the data collected. The ranges are reproduced here to give an idea about the magnitudes expected from such circuits.

Table 2: Signal ranges.

\begin{tabular}{|c|c|c|c|}
\hline Signal & Min Value & Max Value & Peak-Peak \\
\hline $\mathrm{vC} 1$ & $-0.7070 \mathrm{~V}$ & $0.7827 \mathrm{~V}$ & $1.4896 \mathrm{~V}$ \\
\hline $\mathrm{vC} 2$ & $-2.9316 \mathrm{~V}$ & $3.1636 \mathrm{~V}$ & $6.0952 \mathrm{~V}$ \\
\hline $\mathrm{iL}$ & $-41 \mathrm{~mA}$ & $52 \mathrm{~mA}$ & $92 \mathrm{~mA}$ \\
\hline
\end{tabular}

A Fourier analysis was also performed on the three channels. The spectra are quite distinct, with the cutoffs $v_{C 1}=5,700 \mathrm{~Hz}, v_{C 2}=3,200 \mathrm{~Hz}$, and $i_{L}=3,000 \mathrm{~Hz}$. This means that the actual sampling frequency used in the experiment was above the required Nyquist frequency.

\section{Concluding Remarks}

Teaching dynamical systems can be enhanced by simulating and demonstrating the behaviour of various physical systems. Very often in the past, the systems were mechanical, such as the driven pendulum systems from Daedalon Corporation [SmB106], Leybold Didactic, Pasco Scientific, and TELAtomic, Inc. [B1Ba98]. Although the mechanical systems were often expensive and difficult to maintain in the classroom, they provided excellent hands-on experience to the students and researchers [BaB109]. Many alternative systems are electronic, such as the Chua circuit described in this paper. The Chua circuit is easy to implement since it has few components, and can be constructed using commercial off-the-shelf components. Our Chua circuit implementation can demonstrate the entire bifurcation diagram, and can be used to display the strange attractor. The multi-tap microcontroller-driven potentiometer allows the demonstration to be performed quickly and repeatedly. The gained time can then be spent on discussion of the results.

This implementation can also provide the capability to automate the shifts to different states of the Chua circuit.

\section{Acknowledgements}

We would like to thank the Department of Electrical and Computer Engineering at the University of Manitoba for partial financial support of this project.

\section{References}

[Addi97] Paul S. Addison, Fractal and Chaos: An Illustrated Course. Bristol, UK: Institute of Physics Publishing, 1997, 256 pp. \{ISBN 0-7503-0400-6 pbk\} [AlSY96] Kathleen T. Alligood, Tim D. Sauer, and James A. Yorke, Chaos. New York, NY: SpringerVerlag, 1996, 603 pp. \{ISBN 0-387-94677-2 pbk\}

[BaB109] Gregory L. Baker and James A. Blackburn, The Pendulum: A Case Study in Physics. Oxford, UK: Oxford University Press, 2009, 350 pp. \{ISBN: 978-0199557684\}

[B1Ba98] James A. Blackburn and Gregory L. Baker, "A comparison of commercial chaotic pendulums," Am. J. Phys., vol. 66, no. 9, pp. 821-830, Sept 1998. Available as of April 2012 from http://fraden.brandeis.edu/courses/phys39/chaos/Dae dalon\%20AJP.pdf

[BrHT10] H.W. Broer, B. Hasselblatt and F. Takens (eds.), Handbook of Dynamical Systems, Vol. 3, Amsterdam: North-Holland/Elsevier, 2010. \{978-0444-53141-4\}

[Burr99] Burr-Brown, Miniature 2W Isolated Unregulated DC/DC Converters, DDCP0205-15DP Data Sheet. Tuscon, AZ: Burr-Brown Corporation, 1999. Available as of March 30, 2012 from www.datasheetcatalog.org/datasheets/120/193437_D S.pdf

[Chua92] Leon O. Chua, "The genesis of Chua's circuit," Archiv für Elektronik und Übertragungstechnik, vol. 46, no. 4, pp. 250-257, 1992. Available as of March 30,2012 from 
WWW-

inst.eecs.berkeley.edu/ ee129/sp09/handouts/Genesi sChuasCircuit.pdf

[Chua06] Leon O. Chua, "Chua's circuit 10 years later," International Journal of Circuit Theory and Applications, vol. 22, no. 4, pp. 279-305, Jul-Aug 1994. \{DOI: 10.1002/cta.4490220404\}.

[Chua07] Leon O. Chua, "Chua circuit," Scholarpedia, vol 2, no. 10, pp, 1488, 2007.

\{doi:10.4249/scholarpedia.1488\} Available as of March 30, 2012 from http://www.scholarpedia.org/article/Chua_circuit

[ChKM86] Leon O. Chua, Motomasa Komuro, and Takashi Matsumoto, "The double scroll family," IEEE Transactions on Circuits and Systems, vol. 33, no. 11, pp. 1072-1118, Nov 1986.

[ChPW95] Leon O. Chua, Ladislav Pivka, and Chai Wah Wu, "A universal circuit for studying chaotic phenomena," Phil. Trans. R. Soc. Lond. A, vol. 353, pp. 65-84, 16 Oct, 1995.

[Cros03] Michael Cross, "Chua’s cicuit," 2003. Available as of March 30, 2012 from http://www.cmp.caltech.edu/ moc/chaos_new/Chua. html

[Deva92] Robert L. Devaney, A First Course in Chaotic Dynamical Systems: Theory and Experiment. Boston, MA: Addison-Wesley, 1992, 302 pp. \{ISBN 0-20155406-2\}

[ElKe00] A. S. Elwakil and Michael P. Kennedy, "Improved implementation of Chua's chaotic oscillator using the current feedback op-amp," IEEE Trans. Circuits Syst. I: Fundamental Theory and Applications, vol. 47, no. 1, pp. 76-79, 2000.

[HoHi89] Paul Horowitz and Winfield Hill, The Art of Electronics. Cambridge, UK: Cambridge University Press, 1989 (2nd ed.), 1152 pp. \{ISBN 978$0521370950\}$

[Kapi02] Tomasz Kapitaniak, Chaos for Engineers. New York, NY: Springer, 2002 ( $2^{\text {nd }}$ ed.), 142 pp. \{ISBN 3-540-66574-9 pbk\}

[KeRS00] Michael Peter Kennedy, Riccardo Rovatti, and Gianluca Setti, Chaotic Electronics in Telecommunications. Boca Raton, FL: CRC Press, 2000, 445 pp. \{ISNBN 0-8493-2348-7\}

[Kenn92] Michael Peter Kennedy, "Robust op-amp realization of Chua's circuit," Frequenz, vol. 46, no. 3-4, pp. 66-80, Mar-Apr 1992. Available as of March 30,2012 from
http://nonlinear.eecs.berkeley.edu/chaos/RobustOpA mpRealizationOfChuaCircuit.pdf

mpk@midir.ucd.ie

[Kins12] W. Kinsner, Fractal \& Chaos Engineering. Lecture Notes. Winnipeg, Manitoba: University of Manitoba, 2012, 941 pp.

[MaSi05] Ganesh B. Maganti and Sahjendra N. Singh, "Modular adaptive control of chaos in Chua's circuit," International Journal of Bifurcation \& Chaos in Applied Sciences \& Engineering, vol. 15, no. 9, pp. 2973-2984, Sep 2005.

[Math12] MathWorks, MATLAB and Simulink. Natick, MA: MathWorks, 2012. Available as of March 30, 2012 from www.mathworks.com/

[Maxi10] Maxim, 10-Bit, MAX5481, MAX5482, MAX5483, MAX5484 Nonvolatile, Linear-Taper Digital Potentiometers, Data Sheet. Sunnyvale, CA: Maxim Integrated Products, 2010. Available as of March 30, 2012 from http://www.maximic.com/datasheet/index.mvp/id/4730

[Micr03] Microchip, PIC16F876A Microcontroller, Data Sheet. Chandler, AZ: Microchip Technology, 2003, 232 pp.

[NaIn05] National Semiconductor, DAQCard-6062E 12bit Multifuction I/O PCMCIA (PC Card) Family Specifications. Austin, TX: National Semiconductor, Dec 2005. Available as of March 30, 2012 from www.ni.com/pdf/manuals/370724c.pdf

[NaIn08] National Instruments, Multisim (Professional Edition, 30-day trial version). Austin, TX: February 2008. Available as of March 30, 2012 from http://www.ni.com/multisim/

[NaSe03] National Semiconductor, LMC6482AIN CMOS Dual Rail-To-Rail Input and Output Operational Amplifier, Data Sheet DS011713.

National Semiconductor, Sep 2003. Available as of March 30, 2012 from http://nonlinear.eecs.berkeley.edu/chaos/LMC6482.p df

[SmB106] H.J.T. Smith and James A. Blackburn, EM-52 Chaotic Pendulum User's Guide. Salem, MA: Daedalon Corporation, 1998. 34 pp. Available as of April 2012 from

http://fraden.brandeis.edu/courses/phys39/chaos/EM52\%20Daedalon\%20Manual.pdf
CEEA Conf. 2012; Paper 096

Winnipeg, MB; June 17-20, 2012
-9 of $10-$

ChuaChaos-Full-v40.docx

April 6, 2012 
[Spro00a] J.C. Sprott, "Simple chaotic systems and circuits," Am. J. Physics, vol. 68, no. 8, pp. 758-763, Aug 2000.

[Spro00b] J.C. Sprott, "A new class of chaotic circuit," Physics Letters A, vol. 266, pp. 19-23, 14 Feb 2000. Available as of March 30, 2012 from http://sprott.physics.wisc.edu/pubs/paper244.htm

[Ogor97] Maciej J. Ogorzalek, Chaos and complexity in nonlinear electronic circuits. Singapore: World Scientific, 1997, 276pp. \{ISBN 981-02-2873-2\}

[Ott93] Edward Ott, Chaos in Dynamical Systems. Cambridge, UK: Cambridge University Press, 1993, 385 pp. \{ISNBN 0-521-43799-7 pbk\}

[OtSY94] Edward Ott, Tim Sauer, and James A. Yorke (eds.), Coping with Chaos: Analysis of Chaotic Data and the Exploitation of Chaotic Systems. New Yor, NY: Wiley, 1994, 418 pp. \{ISNBN 0-471-02556-9\}

[PaCh89] Thomas S. Parker and Leon O. Chua, Practical Numerical Algorithms for Chaotic Systems. New York, NY: Springer, 1989, 346 pp. \{ISBN 0387-96688-9\}

[PeJS92] H.-O. Peitgen, H. Jurgens and D. Saupe, Chaos and Fractals: New Frontiers of Science. New York (NY): Springer Verlag, 1992 ( $1^{\text {st }}$ ed.), 984 pp.; 2004 ( $2^{\text {nd }}$ ed.), 864 pp. \{ISBN 0-387-20229-3\}

[RuTa71] F. Takens, F. and D. Ruelle, "On the nature of turbulence," Communications in Mathematical
Physics, vol. 20 , pp. 167 - 192, 1971; and vol. 23, pp. 343-344, 1971.

[SaYC91] Tim Sauer, James A. Yorke, and Martin Casdagli, "Embedology," Journal of Statistical Physics, vol. 65, no. 3-4, pp. 579-616, 1991. \{doi:10.1007/BF01053745\}.

[SeSm09] Adel S. Sedra and Kenneth C. Smith, Microelectronics Circuits. Oxford, UK: Oxford University Press, 6th edition, 2009 (6th ed.), 1456 pp. \{ISBN-13: 978-0195323030\}

[Strog94] Steven H. Strogatz, Nonlinear Dynamics and Chaos: With Applications to Physics, Biology, Chemistry, and Engineering. Cambridge MA: Westview / Perseus Books Group, 1994, 498 pp. \{ISBN 0-7382-0453-6 pbk\}

[Take81] Floris Takens, "Detecting strange attractors in turbulence," in D. A. Rand and L.-S. Young (eds.), Dynamical Systems and Turbulence, Lecture Notes in Mathematics, vol. 898 (Warwick 1980). New York, NY: Springer-Verlag. pp. 366-381, 1981.

[Take10] F. Takens, "Reconstruction theory and nonlinear time series analysis," in [BrHT10], pp. 345-378, 2010.

[Will97] Garnett P. Williams, Chaos Theory Tamed. Washington, DC: Joseph Henry Press, 1997, 499 pp. \{ISBN 0-309-06351-5\}
CEEA Conf. 2012; Paper 096

Winnipeg, MB; June 17-20, 2012 